\title{
Expanding Clinical Spectrum of Female X-linked Lymphoproliferative Syndrome 2
}

\author{
Shruthi Suryaprakash ${ }^{1}$, Mohammad El-Baba $^{1}$, Kelly Walkovich $^{2}$, and Sureyya Savasan ${ }^{3}$ \\ ${ }^{1}$ Children's Hospital of Michigan \\ ${ }^{2} \mathrm{C}$ S Mott Children's Hospital \\ ${ }^{3}$ Wayne State University School of Medicine
}

June 1, 2020

Expanding Clinical Spectrum of Female X-linked Lymphoproliferative Syndrome 2

Shruthi Suryaprakash, MD ${ }^{1}$, Mohammad El-Baba ${ }^{2}$, MD, Kelly J. Walkovich, MD ${ }^{3}$, Süreyya Savaşan, MD ${ }^{4}$

${ }^{1}$ Children's Hospital of Michigan

${ }^{2}$ Division of Gastroenterology, Children's Hospital of Michigan

${ }^{3}$ Division of Hematology/Oncology, Immuno-Hematology Comprehensive Program, C.S. Mott Children's Hospital, University of Michigan

${ }^{4}$ Division of Hematology/Oncology and Blood and Marrow Transplant Program, Children's Hospital of Michigan, Carman and Ann Adams Department of Pediatrics, Barbara Ann Karmanos Cancer Center, Central Michigan University College of Medicine

Correspondence: Süreyya Savaşan, MD

3901 Beaubien Blvd.

Division of Hematology/Oncology

Blood and Marrow Transplant Program

Children's Hospital of Michigan

Detroit, Michigan 48201

E-mail: savas1s@cmich.edu

Phone: 313-745-5516 Fax: 313-745-5237

Text word count: 495

Reference count: 4

Tables and figures: 1

Short running title: Spectrum of Symptomatic Female XLP2

Key words: Female XLP2, EBV reactivation, Vitamin B12 deficiency, B-cell lymphopenia, clonal T-LGL proliferation

Dear Editor: 
X-linked lymphoproliferative syndrome type 2 (XLP2) due to pathogenic variants in the X-linked inhibitor of apoptosis (XIAP) gene is a rare cause of primary immunodeficiency. Symptomatic patients, primarily males, present with hemophagocytic lymphohistiocytosis (HLH), inflammatory bowel disease (IBD) and/or transient hypogammaglobinaemia. ${ }^{1}$ However, XLP2 in female patients is complicated with the rarity of symptomatic cases and clinical heterogeneity. ${ }^{2}$ We report a female affected by XLP2 with previously unreported findings.

A currently 18-year-old female presented with fever, abdominal pain, diffuse lymphadenopathy, splenomegaly, and pancytopenia three years ago. She was diagnosed with HLH, treated with steroids and found to have low B-cells and borderline hypogammaglobinemia. Additionally, a single pathogenic variant in XIAP (c.389_392delACAG [p.Asp130Glyfs*11]) was identified. Further workup showed presence of EBV IgG, and normal expression of XIAP protein in only 8-19\% of various white blood cell types by flow cytometry indicating skewed X chromosome inactivation. She had intermittent infections, one resulting in an additional HLH flare with elevated IL-18 and CXCL9 levels that was treated with steroids and intravenous immunoglobulin (IVIG). Repeated EBV PCR testing had been negative.

However, she was found to have EBV reactivation with positive EBV VCA-IgM, high titer VCA-IgG and EA-IgG levels while EBV-PCR was negative when she presented to our clinic with diarrhea. There was ongoing history of headaches, abdominal pain, joint pain, and ADHD at that time. Later, she underwent work up for recurrent abdominal pain, diarrhea, urgency and elevated fecal calprotectin. MRI-enterography and capsule endoscopy were negative. Endoscopy was remarkable for chronic active proctitis. She was prescribed mesalamine with significant improvement in abdominal pain and resolution of mucuosy stools.

Due to persistent knee/ankle pain, she was investigated for peripheral neuropathy and was found to have low vitamin B12 levels (112-145pg/mL; N:180-914) without dietary restrictions, absent anti-intrinsic factor antibodies and negative family history. Her pain improved significantly on vitamin B12 injections and gabapentin with normalization of vitamin B12 levels.

She continued to have fluctuating and borderline low levels of serum immunoglobulins with persistently low B-cells. She was given IVIG supplementation when serum IgG levels were low. No additional HLH flares have occurred. Mild increase in CD5-dim T-cells (9\%) representing T-large granular lymphocytes (T-LGL) and clonal T-cell receptor (TCR) rearrangement pattern were identified in peripheral blood. She continues to have migraine episodes and very high EA-IgG at $>150 \mathrm{U} / \mathrm{mL}(\mathrm{N}<9)$.

Female carriers are at risk for extra hematopoietic manifestations, if they have an extremely skewed $\mathrm{X}$ chromosome inactivation. ${ }^{3}$ She was EBV-PCR negative, but EBV VCA-IgM positive repeatedly suggesting a recent reactivation at presentation to our institution. Persistent high EBV EA-IgG titers is suggestive of ongoing EBV challenge due to immune deficiency and emphasizes the significance of EBV serology testing. Clonal T-LGL expansion may be related to EBV and/or immune deficiency. ${ }^{4}$ Low vitamin B12 raises the possibility of impairment in absorption; the presence of proctitis raises possible subclinical inflammation in the distal ileum. Observed conditions in this case add to the spectrum of this rare entity (Table1).

\section{References}

1. Aguilar C, Latour S. X-linked Inhibitor of Apoptosis Protein Deficiency: More than an X-linked Lymphoproliferative Syndrome. Journal of Clinical Immunology . 2015;35:331-338.

2. Dziadzio M, Ammann S, Canning C, et al. Symptomatic Males and Female Carriers in a Large Caucasian Kindred with XIAP Deficiency. Journal of Clinical Immunology . 2015;35:439-444.

3. Holle JR, Marsh RA, Holdcroft AM, et al. Hemophagocytic lymphohistiocytosis in a female patient due to a heterozygous XIAP mutation and skewed X chromosome inactivation. Pediatric Blood 8 Cancer . 2015;62:1288-1290.

4. Savaşan S, Al-Qanber B, Buck S, Wakeling E, Gadgeel M. Clonal T-cell large granular lymphocyte proliferations in childhood and young adult immune dysregulation conditions. Pediatric Blood \&5 Cancer . $2020 ; 67(5)$. 


\section{Hosted file}

Table1.docx available at https://authorea.com/users/328340/articles/455682-expandingclinical-spectrum-of-female-x-linked-lymphoproliferative-syndrome-2 